\title{
FIXED POINT THEOREMS AND THEIR APPLICATIONS
}


This page intentionally left blank 


\section{FIXED POINT THEOREMS AND THEIR APPLICATIONS}

\section{Ioannis Farmakis Martin Moskowitz}

City University of New York, USA 
Published by

World Scientific Publishing Co. Pte. Ltd.

5 Toh Tuck Link, Singapore 596224

USA office: 27 Warren Street, Suite 401-402, Hackensack, NJ 07601

UK office: 57 Shelton Street, Covent Garden, London WC2H 9HE

\section{British Library Cataloguing-in-Publication Data}

A catalogue record for this book is available from the British Library.

Cover: Image courtesy of Jeff Schmaltz, MODIS Land Rapid Response Team at NASA GSFC.

\section{FIXED POINT THEOREMS AND THEIR APPLICATIONS}

Copyright () 2013 by World Scientific Publishing Co. Pte. Ltd.

All rights reserved. This book, or parts thereof, may not be reproduced in any form or by any means, electronic or mechanical, including photocopying, recording or any information storage and retrieval system now known or to be invented, without written permission from the Publisher.

For photocopying of material in this volume, please pay a copying fee through the Copyright Clearance Center, Inc., 222 Rosewood Drive, Danvers, MA 01923, USA. In this case permission to photocopy is not required from the publisher.

ISBN 978-981-4458-91-7 


\section{Contents}

Preface and Acknowledgments ix

$\begin{array}{ll}\text { Introduction } & 1\end{array}$

1 Early Fixed Point Theorems 3

1.1 The Picard-Banach Theorem . . . . . . . . . . . . 3

1.2 Vector Fields on Spheres . . . . . . . . . . . . 5

1.3 Proof of the Brouwer Theorem and Corollaries . . . . 9

1.3.1 A Counter Example . . . . . . . . . . . 12

1.3.2 Applications of the Brouwer Theorem . . . . . 15

1.3.3 The Perron-Frobenius Theorem . . . . . . . . 16

1.3.4 Google; A Billion Dollar Fixed Point Theorem . . 21

1.4 Fixed Point Theorems for Groups of Affine Maps of $\mathbb{R}^{n} \quad 24$

1.4.1 Affine Maps and Actions . . . . . . . . . . . 25

1.4.2 Affine Actions of Non Compact Groups . . . . . . 30

2 Fixed Point Theorems in Analysis 35

2.1 The Schaüder-Tychonoff Theorem . . . . . . . . 36

2.1.1 Proof of the Schaüder-Tychonoff Theorem . . . . 38

2.2 Applications of the Schaüder-Tychonoff Theorem . . . . 43

2.3 The Theorems of Hahn, Kakutani and Markov-Kakutani 46

2.4 Amenable Groups . . . . . . . . . . . . . . . . 51

2.4.1 Amenable Groups . . . . . . . . . . . . . . 52

2.4.2 Structure of Connected Amenable Lie Groups . . 54 
3 The Lefschetz Fixed Point Theorem 57

3.1 The Lefschetz Theorem for Compact Polyhedra . . . . . 58

3.1.1 Projective Spaces . . . . . . . . . . . . 62

3.2 The Lefschetz Theorem for a Compact Manifold . . . . 64

3.2.1 Preliminaries from Differential Topology . . . . . 64

3.2 .2 Transversality . . . . . . . . . . . . . 67

3.3 Proof of the Lefschetz Theorem . . . . . . . . . . 76

3.4 Some Applications . . . . . . . . . . . . . . . 81

3.4.1 Maximal Tori in Compact Lie Groups . . . . . . 83

3.4.2 The Poincaré-Hopf's Index Theorem . . . . . . . 87

3.5 The Atiyah-Bott Fixed Point Theorem . . . . . . . . . . 94

3.5.1 The Case of the de Rham Complex . . . . . . . . 104

4 Fixed Point Theorems in Geometry 109

4.1 Some Generalities on Riemannian Manifolds . . . . . . . 110

4.2 Hadamard Manifolds and Cartan's Theorem . . . . . . . 124

4.3 Fixed Point Theorems for Compact Manifolds . . . . . . 135

5 Fixed Points of Volume Preserving Maps 143

5.1 The Poincaré Recurrence Theorem . . . . . . . . . . . . 143

5.2 Symplectic Geometry and its Fixed Point Theorems . . 146

5.2.1 Introduction to Symplectic Geometry . . . . . . . 146

5.2.2 Fixed Points of Symplectomorphisms . . . . . . . 153

5.2 .3 Arnold's Conjecture . . . . . . . . . . . . . 154

5.3 Poincaré's Last Geometric Theorem . . . . . . . . . . . 155

5.4 Automorphisms of Lie Algebras . . . . . . . . . . . 163

5.5 Hyperbolic Automorphisms of a Manifold . . . . . . . . 167

5.5.1 The Case of a Torus . . . . . . . . . . . . 169

5.5.2 Anosov Diffeomorphisms . . . . . . . . . . 173

5.5.3 Nilmanifold Examples of Anosov Diffeomorphisms 177

5.6 The Lefschetz Zeta Function _ . . . . . . . . . . . . . . 179

6 Borel's Fixed Point Theorem in Algebraic Groups 187

6.1 Complete Varieties and Borel's Theorem . . . . . . . . . 187

6.2 The Projective and Grassmann Spaces . . . . . . . . . . 190

6.3 Projective Varieties . . . . . . . . . . . . . 193 
6.4 Consequences of Borel's Fixed Point Theorem . . . . . . 197

6.5 Two Conjugacy Theorems for Real Linear Lie Groups . 200

7 Miscellaneous Fixed Point Theorems 203

7.1 Applications to Number Theory . . . . . . . . . . . 203

7.1.1 The Little Fermat Theorem . . . . . . . . . . . 203

7.1.2 Fermat's Two Squares Theorem . . . . . . . . . 205

7.2 Fixed Points in Group Theory . . . . . . . . . . . . 207

7.3 A Fixed Point Theorem in Complex Analysis . . . . . . 209

8 A Fixed Point Theorem in Set Theory 211

$\begin{array}{ll}\text { Afterword } & 217\end{array}$

$\begin{array}{ll}\text { Bibliography } & 219\end{array}$

$\begin{array}{ll}\text { Index } & 229\end{array}$ 
This page intentionally left blank 


\section{Preface and Acknowledgments}

Our intention here is to show the importance, usefulness and pervasiveness of fixed point theorems in mathematics generally and to try to do so by as elementary and self contained means as possible. The book consists of eight chapters.

Chapter 1, Early fixed point theorems, deals mostly with matters connected to the Brouwer fixed point theorem and vector fields on spheres, the Contraction Mapping Principle and affine mappings of finite dimensional spaces. As we shall see, the Brouwer theorem can be generalized in basically two ways, one leading to the Lefschetz fixed point theorem in topology (whose proof is independent of Brouwer) and the other to the Schaüder-Tychonoff fixed point theorem of analysis (which depends on the Brouwer theorem). We shall draw a number of consequences of Brouwer's theorem: among them Perron's theorem concerning eigenvalues of a "positive" operator, which itself has interesting applications to the modern world of computing such as in Google addresses and ranking in professional tennis.

Chapter 2, Fixed point theorems in Analysis, deals with the theorems of Schaüder-Tychonoff, as well as the those of F. Hahn, Kakutani and Kakutani-Markov involving groups of affine mappings, the latter two leading naturally to a discussion of Amenable groups.

Chapter 3 concerns Fixed point theorems in Topology, particularly the Lefschetz fixed point theorem, the H. Hopf index theorem and some of their consequences. As a further application we prove the conjugacy 
theorem for maximal tori in a compact connected Lie group. In addition, we explain the Atiyah-Bott fixed point theorem and its relationship to the classical Lefschetz theorem.

Chapter 4, Fixed point theorems in Geometry, is devoted first to the fixed point theorem of E. Cartan on compact groups of isometries of Hadamard manifolds and then to fixed point theorems for compact manifolds, when the curvature is negative, due to Preissmann, and positive, due to Weinstein.

Chapter 5 concerns Fixed points of maps preserving a volume form, which are important in dynamical systems. We begin with the Poincaré recurrence theorem and deal with some of its philosophical implications. Then we turn to symplectic geometry and fixed point theorems of symplectomorphisms. Here we discuss Arnold's conjecture, prove Poincaré's last geometric theorem and derive a classical result concerning billiards. We then turn to hyperbolic automorphisms of a compact manifold, particularly of a torus, and then to Anosov diffeomorphisms and the analogous Lie algebra automorphisms and explain their significance in dynamics. We conclude this chapter with the Lefschetz zeta function and its many applications.

Chapter 6 deals with the Fixed point theorem of A. Borel for a solvable algebraic group acting on a complex projective variety, the most important such varieties being the Grassmann and flag varieties. We then present some consequences of these ideas. A final section concerns a fixed point theorem giving rise to a conjugacy theorem for unipotent subgroups of real reductive linear Lie groups.

We conclude with two brief chapters. Chapter 7 deals with some connections of fixed points to number theory, group theory and complex analysis, while in Chapter 8, A fixed point theorem in Set Theory, we prove Tarski's fixed point theorem and, as a consequence, the SchröderCantor-Bernstein theorem.

As the reader can see, fixed point theorems are to be found throughout mathematics. These chapters can, for the most part, be read independently. Thus the reader has many options to follow his or her particular interests. 
We thank Oleg Farmakis for his help in creating the diagrams and Hossein Abbaspour for reading an earlier draft of the manuscript and making a number of useful suggestions for improvements. Of course, any mistakes are the responsibility of the authors. Finally, we thank Konstantina and Anita for their patience.

New York, January 2013

Ioannis Farmakis, Martin Moskowitz 